\title{
Structural insights of homotypic interaction domains in the ligand-receptor signal transduction of tumor necrosis factor (TNF)
}

\author{
Young-Hoon Park ${ }^{1, \#}$, Mi Suk Jeong ${ }^{1, \#} \mathcal{E}$ Se Bok Jang ${ }^{1,2, *}$ \\ ${ }^{1}$ Department of Molecular Biology, College of Natural Sciences, Pusan National University, ${ }^{2}$ Genetic Engineering Institute, Pusan National \\ University, Busan 46241, Korea
}

\begin{abstract}
Several members of tumor necrosis factor receptor (TNFR) superfamily that these members activate caspase-8 from death-inducing signaling complex (DISC) in TNF ligand-receptor signal transduction have been identified. In the extrinsic pathway, apoptotic signal transduction is induced in death domain (DD) superfamily; it consists of a hexahelical bundle that contains 80 amino acids. The DD superfamily includes about 100 members that belong to four subfamilies: death domain (DD), caspase recruitment domain (CARD), pyrin domain (PYD), and death effector domain (DED). This superfamily contains key building blocks: with these blocks, multimeric complexes are formed through homotypic interactions. Furthermore, each DD-binding event occurs exclusively. The DD superfamily regulates the balance between death and survival of cells. In this study, the structures, functions, and unique features of DD superfamily members are compared with their complexes. By elucidating structural insights of DD superfamily members, we investigate the interaction mechanisms of DD domains; these domains are involved in TNF ligand-receptor signaling. These DD superfamily members play a pivotal role in the development of more specific treatments of cancer. [BMB Reports 2016; 49(3): 159-166]
\end{abstract}

\section{INTRODUCTION}

Apoptotic cell death is a critical decision point in the life cycle of mammalian cells. It is triggered by intrinsic, mitochondria-mediated or extrinsic receptor-mediated signaling pathways (1). This process occurs via a well-defined sequence of

${ }^{*}$ Corresponding author. Tel: +82-51-510-2523; Fax: +82-51-5812544; E-mail: sbjang@pusan.ac.kr

${ }^{\text {"}}$ These authors contributed equally to this work.

http://dx.doi.org/10.5483/BMBRep.2016.49.3.205

Received 8 October 2015, Revised 4 November 2015, Accepted 24 November 2015

Keywords: Apoptosis, DD, DED, TNF morphological events (2). The intracellular mechanism that is responsible for apoptosis appears to be similar in almost all mammalian cells. These mechanisms depend on the members of a protease superfamily, which has cysteine at their enzyme's activity site. The substrates cleave at specific aspartic acids. Hence, they are termed caspases (3). During this process, the dying cell undergoes condensation of nucleus and cytoplasm. Furthermore, blebs develop in the plasma membrane. The cell breaks up into membrane-enclosed fragments that are known as apoptotic bodies; these apoptotic bodies contain intact organelles. The apoptotic bodies are rapidly engulfed by neighboring cells or professional phagocytes, such as dendritic cells and macrophages. This prevents the release of potentially toxic chemicals in tissues $(1-2,4)$.

Biological responses may vary from cell survival to cell death. These responses are mediated by many protein complexes that contain homotypic interaction motifs, such as death ligand/receptor complex, apoptosome protein complex, and DISC $(5,6)$. The typical model of signal transduction pathways entails transmembrane receptors. These receptors become active after docking a ligand. Then, they transmit signals in the cytoplasm to generate new signal transduction complexes $(5,6)$. Owing to the interaction between tumor necrosis factor- $\alpha(\mathrm{TNF}-\alpha)$ and tumor necrosis factor receptor type 1 (TNFR-1), there is rapid clustering and internalization of death domain (DD) complex. This process proceeds through the formation of clathrin-coated endocytic vesicles (7). After the internalization of TNFR-1 in human endothelial cells, DD complex induces NF- $\kappa B$ regulation factor. However, TNFR-1 is able to promote apoptotic cell death. The DD superfamily induces cell survival and apoptotic cell death via TNFR-1 dependent signal cascade. This superfamily is a vital regulator for maintaining the homeostasis of cells in humans (Fig. 1) (5-9).

In the extrinsic apoptosis pathway, the interaction between DD superfamily members plays an important role in the formation of DISC. With this pathway, procaspase-8 is activated (10). The DISC is assembled in the cytoplasm. Furthermore, TNF-related apoptosis inducing ligand (TRAIL), TNF-related weak inducer of apoptosis (TWEAK), TNF- $\alpha$, TNF- $\beta$, and Fas ligand (FasL) are the death ligands that interact with death re-

ISSN: 1976-670X (electronic edition)

Copyright (C) 2016 by the The Korean Society for Biochemistry and Molecular Biology

(c) This is an open-access article distributed under the terms of the Creative Commons Attribution Non-Commercial License (http://creativecommons.org/licenses/by-nc/4.0) which permits unrestricted non-commercial use, distribution, and reproduction in any medium, provided the original work is properly cited. 


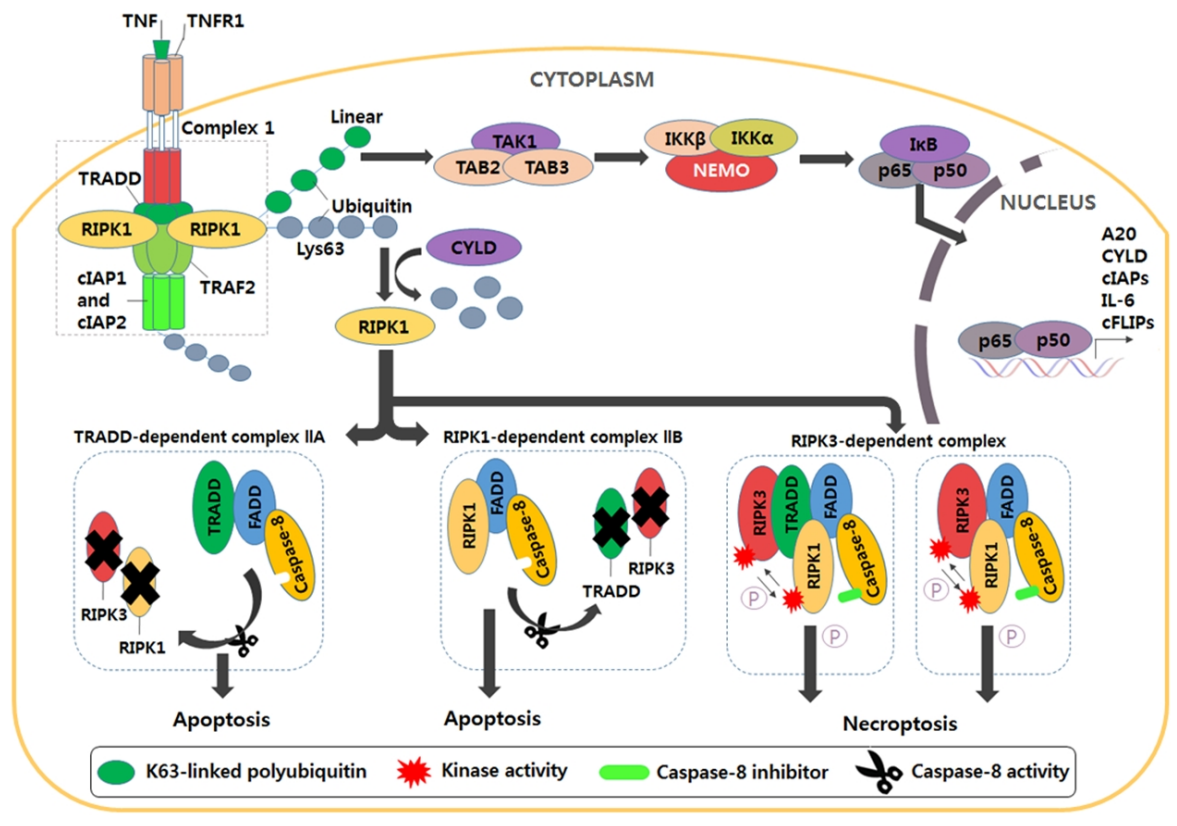

Fig. 1. Cell signaling pathway through which DD complex elicits a balance between survival and programmed cell death (9). ceptors and TNF receptors. They constitute apoptotic signaling platforms of extrinsic pathway $(5,10,11)$.

Apoptotic signal transduction is induced through a homology domain containing a hexahelical bundle of 80 amino acids. With this process, DD superfamily members are produced (12-14). Furthermore, DDs construct key building blocks that are involved in the formation of multimeric complexes; these complexes are associated with death signaling cascades. In this study, we summarize recent findings that elucidate three dimensional structures of TNF ligand-receptor superfamily. They provide molecular and functional characterization of homotypic DD interaction motifs, which are associated with programmed cell death.

\section{THE DEATH-FOLD INTERACTIONS}

When the signal of Fas receptor is activated, the Fas-associated death domain protein (FADD) develops homotypic interaction motifs, such as DD and death effector domain (DED). With these motifs, caspase- 8 can be recruited to the docking site. In this process, Fas and caspase-8 interact simultaneously via DD and DED (9). The aspartate-specific cysteine proteases (Caspases) are primary executioners of non-inflammatory cell death. Effector caspases cleave regulatory enzymes, such as poly (ADP-ribose) polymerase (PARP). They also cleave activating endonucleases, such as caspase-activated deoxyribonuclease (CAD) (15). Biologically, caspases are broadly categorized into initiator and effector caspases. The initiator caspases have death-fold motifs, such as DED or caspase recruitment domain (CARD). They trigger non-inflammatory cell death by activating effector caspases. The activation of effectors is conducted by initiator caspases, which act at cytoplasmic sites, nucleus, and other organelles. Many death-fold interactions are formed by specific adaptor molecules, such as CARDs, DEDs, DDs, and pyrin domains (PYDs): they belong to the superfamily of DD (16).

An apoptotic protease cascade develops when cytochrome c influences dATP-dependent interaction of apoptotic protease activating factor (Apaf-1) and caspase-9. Consequently, the apoptosome is generated via a CARD-CARD homotypic interaction (Fig. 2A-C) (17). When cytochrome $\mathrm{c}$ and Apaf-1 are bound to each other, the signaling complex is activated. Consequently, there is autoactivation and recruitment of procaspase 9 (18). The active apoptosome complex contains several Apaf-1, procaspase-9 proteins, and general complex forms; the molecular weight of these components is approximately $700 \mathrm{kDa}(19)$.

\section{THE DD SUPERFAMILY}

Many proteins have significantly different amino acid sequences. However, functional and structural characteristics indicate that a common ancestor is probably involved in superfamilies (20). The DD superfamily, which is characterized by the presence of conserved homotypic interaction motifs, has emerged as the principal mediator in the transduction of a cell signal (21). About 100 members of DD, DED, CARD, and PYD subfamilies have been identified till date (12-14). Despite the appreciable divergence in the overall sequence of each member, the structure of each DD is hallmarked. The DD superfamily constitutes six amphipathic $\alpha$-helices that are folded in an antiparallel $\alpha$-helical bundle. The atomic structures of DD superfamily are determined by either nuclear magnetic reso- 

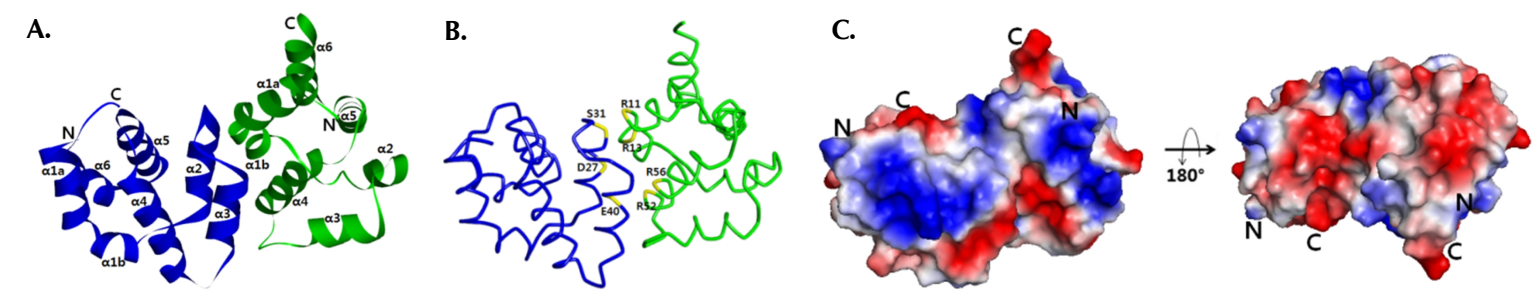

D.

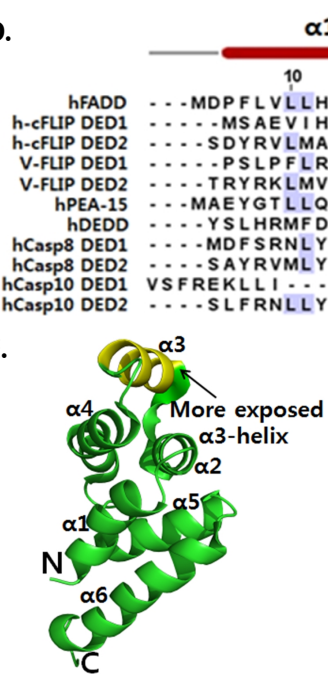

$\alpha 1$

$\alpha 2$
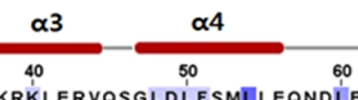

$\alpha 5$

80

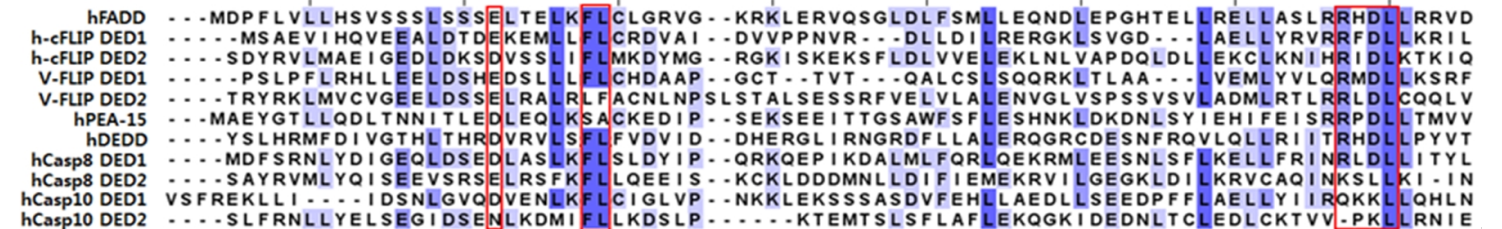

E.

F.

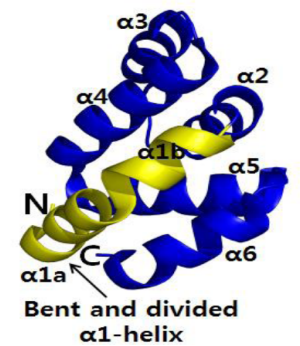

G. Extended $\alpha 2 / \alpha 3$ flexible region More short

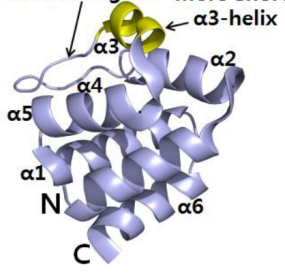

H.

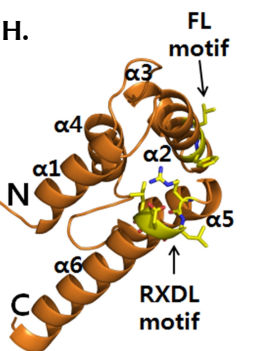

Fig. 2. (A) Ribbon representations of Apaf-1 (blue) and caspase-9 (green). CARDs complex is shown (PDB ID: 3YGS). (B) Carbon stick representation of CARDs complex is shown. Each important amino acid in CARD interaction is shown in yellow. (C) The Apaf-1 and caspase-9 complex, which undergoes a $180^{\circ}$ rotation along the horizontal axis, is shown as a surface representation. (D) Amino acid sequence alignment of DED superfamily members. The secondary structural elements of DED superfamily members, as indicated. Alpha helices are presented as red ellipses, while loop regions are presented as gray lines. The negatively charged amino acids are conserved through species, while highly conserved motifs are presented in red. (E-H). Three dimensional structures of different DD subfamilies (23). (E) The DD of FADD (PDB: 1E3Y). (F) The CARD of Apaf-1 (PDB: 1CY5). (G) The pyrin domain of apoptosis repressor with CARD protein (PDB: 1UCP). (H) The second DED of v-FLIP (PDB: 2BBR). The DD superfamily structures are presented as flat ribbon. Each specific region is illustrated (yellow).

nance (NMR) or X-ray crystallography (Fig. 2E-H) (22-26).

Recently, many studies have been conducted on DD superfamily proteins. In these studies, it is proved that DD superfamily proteins contain many different motifs; the features of these different motifs have significant differences. The differences exist even within the members of subfamilies. These differences are caused by the alterations in the size and arrangement of $\alpha$-helices. Furthermore, these differences are also caused by the varying distribution of charged and hydrophobic residues along the surface (23-26). As a result, all the superfamily members are included in the homotypic interaction proteins of every kind. Furthermore, they do not cross the boundaries of subgroup (27). Compared to respective structural features, it is likely that DDs have an exposed third $\alpha$-helix. This $\alpha$-helix is more pliable than other subfamilies (Fig. 2E-H) (22-26). The CARD is another DD subfamily consisting of members with a highly conserved structure. There is striking similarity between members of CARD and the amino-terminal domains of DD-containing proteins: CRADD and Apaf-1 (24,
28). The most significant difference between CARD and other subfamilies is as follows: the first $\alpha$-helix $(\alpha 1)$ leads to the disruption of hydrogen bonds between nitrogen atoms of amide and oxygen atoms in the carbonyl group of CARD. The helix in CARD is composed of two short helices in $\alpha$-helix: the first part is $\alpha 1 \mathrm{a}$ and the second part is $\alpha 1 \mathrm{~b}$. Both the parts are connected by a linker region. Owing to bending, residues present at the C-terminal of helix $\alpha 1$ in Apaf- 1 are closer to the hydrophobic core; they are far away from the corresponding residues in RAIDD CARD $(24,28)$. This helical shift is propagated in the remaining portion of the molecule. Consequently, the positions and boundaries of all other helices and surface loops are altered $(24,28-30)$. The PYD subfamily members have a hydrophobically stabilized loop region between helices $\alpha 2$ and $\alpha 3$. The connecting loop of PYD is longer than that of other subfamilies, whereas the exposed helix $\alpha 3$ in PYD is shortest in the DD superfamily $(25,31)$. The DED subfamily has exposed hydrophobic sites, which are smaller than DDs in the helix region. Moreover, a conserved motif is present between 
helices $\alpha 5$ and $\alpha 6(26,32,33)$. This specific motif contains amino acids, such as aspartic acid and arginine, in helix $\alpha 6$. They mediate protein folding and ensure the functional conformation of DEDs. Negatively charged amino acids (glutamic acid, aspartic acid, or asparagine) are conserved at the N-terminal. Furthermore, there is an Arg-x-Asp-Leu (RxDL; ' $\mathrm{x}$ ' for any amino acid) motif in nearly all DEDs (33). Most single and tandem DED proteins have RxDL motifs that are consistent in the highly conserved region; however, this motif is not present in other DD subfamilies (Fig. 2D) $(26,33,34)$. When the three-dimensional structure of MC159 (v-FLIP) was elucidated, a hydrogen-bonded triad was revealed on the surface of DED1 and DED2. This triad contributes to the highly charged features on one face of the structure (32). Previous studies prove that RxDL motif mediates molluscum contagiosum virus (MCV) protein MC159 and block apoptosis $(33,35)$. Convertsely, the charged amino acids in caspase- 8 and caspase- 10 are either greatly altered or missing in DEDs. The DED2 domain in caspase-8 has three charged amino acids: Glu, Lys, and Ser (36). By converting arginine to lysine, the hydrogen bonding potential decreases and there is interaction with only one negatively charged amino acid. In caspase-10, RxDL motif is missing in both DED1 and DED2. Therefore, we deduce that this motif does not play an important role in the recruitment of caspase. However, it is important in other functions of proteins containing DED (32).

\section{THE DEATH INDUCIBLE SIGNALING COMPLEX}

NMR spectroscopy is used to develop three-dimensional models of FADD, DED, and DD $(32,37)$. Members of DD superfamily include Fas and FADD; they share helix $\alpha 6$ strands with Greek key topology $(32,27)$. It has been shown that this topology governs the process of protein folding. This process is initiated by the formation of a hydrophobic pocket, which contains almost all the conserved sites of DD superfamily (38). The DD domains regulate the assembly of death inducing, multimeric signaling complexes exhibiting the activity of critical effectors (9). However, very limited information is available about the structural features of DDs complexes till date. By performing X-ray crystallography, models of cell death have been identified; these models induced Fas/FADD-DISC (39). The complex activates procaspase-8, which forms an integral part of programmed cell death pathway. It is triggered by binding between Fas-receptor and FasL or between TNFR-1 and TNF- $\alpha(40,41)$. FasL is expressed in activated T lymphocytes or natural killer (NK) cells. They cause the destruction of target cells, such as virus-infected or damaged cells (42). The Fas/ FADD-DISC crystal structure exists in the closed form owing to lack of a stimulus. This is followed by multimerization of its factors; they form an active platform (39). In FasL-treated cells, Fas and FADD proteins form signaling complexes via modular interaction in their C-terminal DDs $(39,42)$. Furthermore, FADD recruits caspase-8 through DED, promoting their enzy- matic activity and stimulating the expression of apoptosis-related proteins $(38,39,43)$.

In Fas receptor signaling, the central event involves the formation of DISC; it is comprised of procaspase-8, procaspase-10, cellular FLICE-like inhibitory proteins (c-FLIP), and adapter molecule FADD (44). The procaspase-mediated apoptosis is crucial to Fas/FADD-DISC signaling study. All procaspase-8 isoforms have two $\mathrm{N}$-terminal DEDs that are similar to the isoforms of c-FLIP protein. In this study, we identified several DED isoforms of procaspase and c-FLIP at DISC. There were two procaspase-8 isoforms: (procaspase-8a [p55] and procaspase-8b [p53]). In addition, there were three c-FLIP isoforms, such as c-FLIP long (c-FLIP $\mathrm{L}_{\mathrm{L}}$, c-FLIP short (c-FLIP $\mathrm{s}_{\mathrm{s}}$, and c-FLIP Raji (c-FLIPR) (Fig. 3) (26, 44-47). Recently, Schleich et al. demonstrated that FADD, procaspase, and c-FLIP were the DED members forming a signaling complex in their DED domains (48). The new paradigm of DISC proved that procaspase- $8 / 10$ is activated via the assembly of DED chains assembly $(48,49)$. These chains enable the homodimerization of procaspase-8, which plays an important role in the activation of procaspase- 8 in DISC. For example, owing to the mutations of some key binding amino acids in procaspase-8, DED2 interrupts the formation of DED chain in cells. Moreover, these specific binding sites are conserved in nearly all DED domains; these domains interact with another DED via hydrophobic interaction motif of phenylalanine/leucine (FL motif) (Figs. 2D and H) $(22-26,49,50)$. After the formation of DED chain, procaspase-8a/b homodimer processing involves two sequential cleavage steps at the DISC. The first cleavage step takes place at the aspartic acid 374, which lies between p18 and p10 caspase subunits. The second cleavage step occurs in two aspartic acids: Asp216 and Asp384. Consequently, large protease subunit $\mathrm{p} 18$, small protease subunit $\mathrm{p} 10$, and the prodomain p26/p24 are produced. Owing to DED chain processing, the active caspase- 8 heterotetramer, $\mathrm{p} 18_{2}-\mathrm{p} 10_{2}$, is subsequently released into cytosol and it triggers an apoptotic signal cascade $(51,52)$. The procaspase-8-dependent apoptotic death is interrupted by c-FLIP; however, it is also activated when DED interacts at DISC. The shorter c-FLIP isoforms (c-FLIP and $\mathrm{c}-\mathrm{FLIP}_{\mathrm{R}}$ ) consist of only tandem DEDs that block cell death and procaspase-8 cleavage. However, the long splice variant of c-FLIP isoform (c-FLIP $\mathrm{L}_{\mathrm{L}}$ ) either accelerates or blocks cell death and procaspase-8 cleavage; the interaction of c-FLIP isoform depends on its density $(53,54)$. The DED chain model helps us in elucidating the molecular mechanisms of DD superfamily.

\section{SIGNAL TRANSDUCTION OF LIGANDS AND RECEPTORS ASSOCIATED WITH TNF SUPERFAMILY}

Two different forms of TNF- $\alpha$ and lymphotoxin- $\alpha$ were identified in activated macrophages and T cells $(55,56)$. Several members of TNF superfamily and their receptors are identified in many tissues and organs of animals (57). Each factor of TNF 
A.
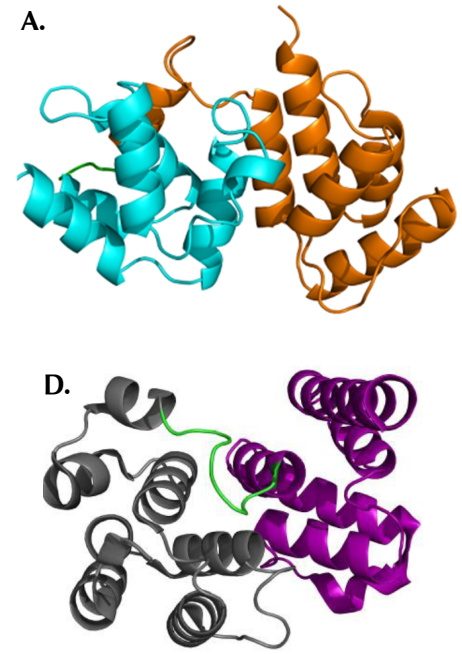

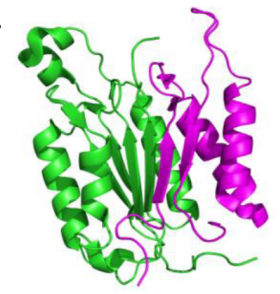

E.

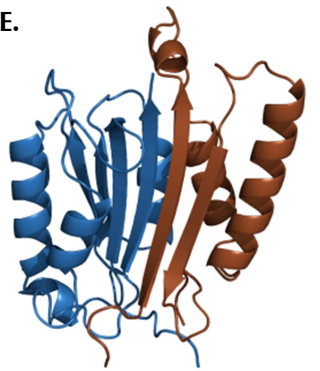

C.

\begin{tabular}{|c|c|c|c|c|}
\hline & \multicolumn{3}{|r|}{ 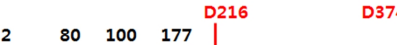 } & 479 \\
\hline & DED1 & DED2 & Large protease & Small protease \\
\hline $1^{\text {st }}$ cleavas & DED1 & DED2 & Large protease & Small protease \\
\hline Ind cleavac & DED1 & DED2 & Large protea & Small prote \\
\hline
\end{tabular}

F.

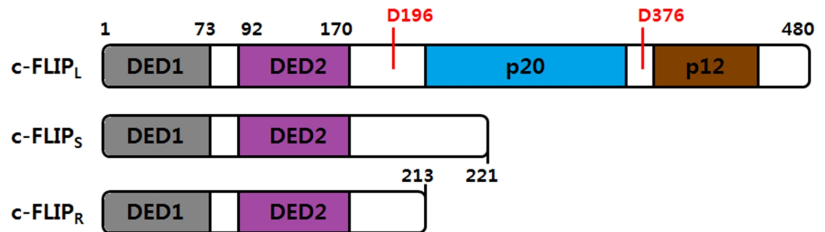

Fig. 3. Three dimensional structures of procaspe-8 and c-FLIP isoforms. (A) The tandem DEDs of procaspase-8 (PDB ID: 4GBW). (B) The subunits of procaspase-8 (p18 and p10) (PDB ID: 4JJ7). (C) The domain structures of procaspase-8 isoforms. (D) Homology model of C-FLIP DEDs (v-FLIP) (PDB ID: 2BBR). (E) The subunits of c-FLIP (p20 and p12) (PDB: 3H11). (F) The domain structures of c-FLIP isoforms.

superfamily interacts with at least one receptor of TNFR superfamily. Furthermore, some TNF factors bind with several receptors $(5,8,21)$. TNF- $\alpha$ is a cytokine that exhibits inflammatory activity; a member of a group of cytokines is included in the TNF-ligand superfamily. The TNF-ligand superfamily plays an important role in regulating cellular life and death $(57,58)$.

TNF- $\alpha$ plays an important role in the regulation of immune cells; however, it can also induce apoptotic cell death and inhibit tumorigenesis, metastasis, bone resorption, and viral replication (59-61). In addition, TNF- $\alpha$ plays an important role in the activation of two distinct cell surface receptors: TNFR-1 and TNFR-2 $(6,59,60)$. The expression of TNFR-2 is manifested in the immune system and endothelial cells, whereas the expression of TNFR-1 is detected in many tissues. In TNFR-1, the extracellular position has four characteristic cysteine-rich domains (CRDs) for the purpose of interaction; these CRDs bind directly with the TNF- $\alpha$ trimer $(6,57-59)$. Previous studies prove that owing to the interaction between TNF- $\alpha$ and TNFR-1, activation of proteins is mediated in the following components: tumor necrosis factor receptor type 1-associated death domain (TRADD), receptor-interacting serine/threonine-protein kinase 1 (RIPK1), and TNF-R-associated factor 2 (TRAF2). These events play a crucial role in the cascade of TNF signal transduction pathway (59-61).

\section{DD SUPERFAMILY-MEDIATED CELL SIGNAL CASCADE}

In most cell lines, nearly all death receptors mediate apoptosis; moreover, TNFR has several signal transduction pathways, controlling a broad spectrum of life and death in cells (Fig. 1) $(9,57,58)$. With an activated TNFR-1 signal cascade, the lysine 63 polyubiquitination of RIPK1 is promoted $(62,63)$. Protein kinase RIPK1 functions as a kinase depending on the extent to which it undergoes ubiquitination. It either regulates prosurvival transcription factor NF- $\mathrm{kB}$ or promotes apoptotic and necrotic cell death under the effect of TRADD, FADD, caspase-8, and RIPK3 (62-65). The assembly of RIPK1, TNFR-1, and TRADD complex (complex I) was found to be inhibited by cellular inhibitor of apoptosis protein 1 (CIAP1) and cellular inhibitor of apoptosis protein 2 (CIAP2) in cytosol $(62,66,67)$. Furthermore, with the interaction of these two inhibitor proteins, the polyubiquitin chains of RIPK1 were formed. They act as a crucial binding site in TAK1/TAB2/3 complex and NEMO/ IKK $\alpha / \beta$ complex. NF- $\kappa B$ is translocated in the nucleus by these two complexes. Thereafter, there is expression of cylindromatosis (CYLD), tumor necrosis factor alpha-induced protein 3 (TNFAIP3), and other NF-KB related factors $(64,66,68)$.

Recent studies prove the intracellular signaling kinase RIPK1 acts as a key switch in the regulation of cell fate. Depending on the cellular context, RIPK1 determines whether the pieotropic cytokine TNF induces activation, apoptosis, or programmed necrosis of NF-kB $(69,70)$. RIPK1 is involved in the regulation of cell death when there is internalization of death receptor. RIPK1 is dissociated from TNFR-1 of TNF signal transduction complex, while lysine 63 deubiquitination of RIPK1 is mediated by the following deubiquitination enzymes: CYLD and A20. These enzymes inhibit the expression of prosurvival transcription factor, NF- $\kappa B(5,62,63)$. Following deubiquitination, RIPK1 interacts with TRADD, FADD, RIPK3, 
generating TRADD-dependent complex (complex IIA) and RIPK1-dependent complex (complex IIB) (63, 70-72). Complex IIA necessitates a TRADD-FADD scaffold to recruit caspase-8, which has DED and DD sites. Thus, an apoptotic pathway is initiated $(5,21,71)$.

When TRADD does not exist, RIPK1 is formed and apoptosis signal pathway is initiated. With the formation of complex IIB, there is FADD-mediated recruitment and activation of caspase- 8 at the cleavage of RIPK1 and RIPK3. Owing to the interaction between these two RIPK superfamily proteins, there is increase in metabolic activities. In this process, the activities of glutamate dehydrogenase 1 , glutamate ammonia ligase, and glycogen phosphorylase may an important regulatory role. This results in programmed necrotic cell death $(63,65,69-71)$.

When X-ray crystallographic and NMR studies were performed on the members of DD superfamily, a great deal of information was obtained. These studies prove that its members play a pivotal role in the assembly and regulation of complexes, which are crucial for cell survival and programmed cell death signaling. They ensure the recruitment of kinase proteins through an interaction with homotypic motifs. Despite conservation in topology, members of DD superfamily have three dimensional structures with significant difference. Among DD superfamily proteins, the major structural difference is the presence of diverse twisting angles between each $\alpha$-helix bundle. These angles are formed by several helices in $\mathrm{DD}$, and they exhibit the biggest difference in three dimensional structure. Owing to the structural differences in DD superfamily, none of the superfamily members undergo cross interactions among subgroups. Owing to the interaction between TNF-ligand and TNF-receptor superfamily, there is mediation in many DD proteins. These proteins create new signal transduction complexes, such as apoptosome, DISC, and other multimers of DD. The apoptosis that is induced by TNF receptor is a biologically relevant form of cell death; it occurs in the presence of sufficient DISC activity. In the extrinsic pathway, Fas/FADD oligomerization regulates apoptotic signal cascade. Depending on the homotypic interaction with DD superfamily, the transition occurs between programmed cell death and survival (71). Previous studies prove that the expression of DD superfamily increases in cancer cells and tissues, which are treated with anticancer agents. This indicates that DD superfamily participates in the action of these agents $(73,74)$. Moreover, many extrinsic signaling pathways stimulate tumor cells to induce programmed cell death; these events are independent of tumor-suppressor p53 (75). Thus, DD receptors might be a useful target in cancer research studies. In this study, we summarized the structural characterization and function of homotypic interaction motifs and their complexes. The findings of this study play an important role in the development of more specific treatments that are used for curing inflammation and cancer.

\section{ACKNOWLEDGEMENTS}

This study was supported by the Basic Science Research Program of the National Research Foundation of Korea (NRF). It was funded by the Ministry of Education, Science, and Technology and (2014-046536) M.S.J. This study was also supported by the Research Fund Program of Research Institute for Basic Sciences, Pusan National University, Korea, 2014. (Project No. RIBS-PNU-2014-01200001).

\section{REFERENCES}

1. Chowdhury I, Tharakan B and Bhat GK (2006) Current concepts in apoptosis: the physiological suicide program revisited. Cell Mol Biol Lett 11, 506-525

2. Rich T, Watson CJ and Wyllie A (1999) Apoptosis: the germs of death. Nat Cell Biol 1, e69-e71

3. Ola MS, Nawaz M and Ahsan H (2011) Role of Bcl-2 family proteins and caspases in the regulation of apoptosis. Mol Cell Biochem 351, 41-58

4. Brown S, Heinisch I, Ross E, Shaw K, Buckley CD and Savill J (2002) Apoptosis disables CD31-mediated cell detachment from phagocytes promoting binding and engulfment. Nature 418, 200-203

5. Wilson NS, Dixit V and Ashkenazi A (2009) Death receptor signal transducers: nodes of coordination in immune signaling networks. Nat Immunol 10, 348-355

6. Zhang JY, Tao S, Kimmel R and Khavari PA (2005) CDK4 regulation by TNFR1 and JNK is required for NF- $\mathrm{KB}$-mediated epidermal growth control. J Cell Biol 168, 561-566

7. Mosselmans R, Hepburn A, Dumont JE, Fiers W and Galand P (1988) Endocytic pathway of recombinant murine tumor necrosis factor in L-929 cells. J Immunol 141, 3096-3100

8. Chinnaiyan AM, O'Rourke K, Yu GL, et al (1996) Signal transduction by DR3, a death domain-containing receptor related to TNFR-1 and CD95. Science 274, 990-992

9. Berghe TV, Linkermann A, Jouan-Lanhouet S, Walczak $H$, and Vandenabeele P (2014) Regulated necrosis: the expanding network of non-apoptotic cell death pathways. Nat Rev Mol Cell Bio 15, 135-147

10. Muzio M, Chinnaiyan AM, Kischkel FC, et al (1996) FLICE, a novel FADD-homologous ICE/CED- 3-like protease, is recruited to the CD95 (Fas/APO-1) death-inducing signaling complex. Cell 85, 817-827

11. Walczak H, Miller RE, Ariail K, et al (1999) Tumoricidal activity of tumor necrosis factor-related apoptosis-inducing ligand in vivo. Nat Med 5, 157-163

12. Tartaglia LA, Ayres TM, Wong GH and Goeddel DV (1993) A novel domain within the $55 \mathrm{kd}$ TNF receptor signals cell death. Cell 74, 845-853

13. Nagata S (1997) Apoptosis by death factor. Cell 88, 355-365

14. Scaffidi C, Medema JP, Krammer PH and Peter ME (1997) FLICE is predominantly expressed as two functionally active isoforms, caspase-8/a and caspase-8/b. J Biol Chem 272, 26953-26958

15. Sakahira H, Enari M and Nagata S (1998) Cleavage of 
CAD inhibitor in CAD activation and DNA degradation during apoptosis. Nature 391, 96-99

16. Muzio M, Stockwell BR, Stennicke HR, Salvesen GS and Dixit VM (1998) An induced proximity model for caspase-8 activation. J Biol Chem 273, 2926-2930

17. Zhou P, Chou J, Olea RS, Yuan J and Wagner G (1999) Solution structure of Apaf-1 CARD and its interaction with caspase-9 CARD: a structural basis for specific adaptor/caspase interaction. Proc Natl Acad Sci U S A 96, 11265-11270

18. Srinivasula SM, Ahmad M, Fernandes-Alnemri $T$ and Alnemri ES (1998) Autoactivation of procaspase-9 by Apaf-1-mediated oligomerization. Mol Cell 1, 949-957

19. Cain K, Brown DG, Langlais C and Cohen GM (1999) Caspase activation involves the formation of the aposome, a large $(\sim 700 \mathrm{kDa})$ caspase-activating complex. J Biol Chem 274, 22686-22692

20. Ortells MO and Lunt GG (1995) Evolutionary history of the ligand-gated ion-channel superfamily of receptors. Trends Neurosci 18, 121-127

21. Ashkenazi A and Dixit VM (1998) Death receptors: signaling and modulation. Science 281, 1305-1308

22. Berglund $H$, Olerenshaw D, Sankar A, Federwisch $M$, McDonald NQ and Driscoll PC (2000) The three-dimensional solution structure and dynamic properties of the human FADD death domain. J Mol Biol 302, 171-188

23. Kersse K, Verspurten J, Berghe TV and Vandenabeele P (2011) The death-fold superfamily of homotypic interaction motifs. Trends Biochem Sci 36, 541-552

24. Vaughn DE, Rodriguez J, Lazebnik $Y$ and Joshua-Tor $L$ (1999) Crystal structure of Apaf-1 caspase recruitment domain: an $\alpha$-helical Greek key fold for apoptotic signaling. J Mol Biol 293, 439-447

25. Liepinsh E, Barbals R, Dahl E, Sharipo A, Staub E and Otting G (2003) The death-domain fold of the ASC PYRIN domain, presenting a basis for PYRIN/PYRIN recognition. J Mol Biol 332, 1155-1163

26. Yang JK, Wang L, Zheng L, Wan F, Ahmed M, Lenardo M. $\mathrm{J}$ and $\mathrm{Wu} \mathrm{H}$ (2005) Crystal structure of MC159 reveals molecular mechanism of DISC assembly and FLIP inhibition. Mol Cell 20, 939-949

27. Park HH, Lo YC, Lin SC, et al (2007) The death domain superfamily in intracellular signaling of apoptosis and inflammation. Annu Rev Immunol 25, 561

28. Qin H, Srinivasula SM, Wu G, Fernandes-Alnemri T, Alnemri ES and Shi Y (1999) Structural basis of procaspase- 9 recruitment by the apoptotic protease-activating factor 1. Nature 399, 549-557

29. Humke EW, Shriver SK, Starovasnik MA, Fairbrother WJ and Dixit VM (2000) ICEBERG: a novel inhibitor of interleukin-1 $\beta$ generation. Cell 103, 99-111

30. Srimathi T, Robbins SL, Dubas RL, Hasegawa M, Inohara $\mathrm{N}$ and Park YC (2008) Monomer/dimer transition of the caspase-recruitment domain of human Nod1. Biochemistry $47,1319-1325$

31. Pinheiro AS, Proell $M$, Eibl $C$, Page R, Schwarzenbacher $R$ and Peti W (2010) Three-dimensional structure of the NLRP7 pyrin domain: insight into pyrin-pyrin-mediated effector domain signaling in innate immunity. J Biol Chem $285,27402-27410$
32. Eberstadt M, Huang B, Chen Z, et al (1998) NMR structure and mutagenesis of the FADD (Mort1) death-effector domain. Nature 392, 941-945

33. Garvey TL, Bertin J, Siegel RM, Wang GH, Lenardo MJ and Cohen JI (2002) Binding of FADD and caspase-8 to molluscum contagiosum virus MC159 v-FLIP is not sufficient for its antiapoptotic function. J Virol 76, 697-706

34. Weber $\mathrm{CH}$ and Vincenz C (2001) A docking model of key components of the DISC complex: death domain superfamily interactions redefined. FEBS Lett 492, 171-176

35. Bertin J, Armstrong RC, Ottilie S, et al (1997) Death effector domain-containing herpesvirus and poxvirus proteins inhibit both Fas-and TNFR1-induced apoptosis. Proc Natl Acad Sci U S A 94, 1172-1176

36. Tibbetts MD, Zheng L and Lenardo MJ (2003) The death effector domain protein family: regulators of cellular homeostasis. Nature Immunol 4, 404-409

37. Huang B, Eberstadt M, Olejniczak ET, Meadows RP and Fesik SW (1996) NMR structure and mutagenesis of the Fas (APO-1/CD95) death domain. Nature 457, 1019-1022

38. Steward A, McDowell GS and Clarke J (2009) Topology is the principal determinant in the folding of a complex all-alpha Greek key death domain from human FADD. J Mol Biol 389, 425-437

39. Scott FL, Stec B, Pop C, et al (2009) The Fas-FADD death domain complex structure unravels signalling by receptor clustering. Nature 457, 1019-1022

40. Taylor RC, Cullen SP and Martin SJ (2008) Apoptosis: controlled demolition at the cellular level. Nat Rev Mol Cell Biol 9, 231-241

41. Strasser A, Jost PJ and Nagata S (2009) The many roles of FAS receptor signaling in the immune system. Immunity 30, 180-192

42. Itoh $\mathrm{N}$ and Nagata S (1993) A novel protein domain required for apoptosis. Mutational analysis of human Fas antigen. J Biol Chem 268, 10932-10937

43. Krammer PH, Arnold R and Lavrik IN (2007) Life and death in peripheral T cells. Nat Rev Immunol 7, 532-542

44. Öztürk S, Schleich K and Lavrik IN (2012) Cellular FLICE-like inhibitory proteins (c-FLIPs): fine-tuners of life and death decisions. Exp Cell Res 318, 1324-1331

45. Shen C, Yue H, Pei J, Guo X, Wang T and Quan JM (2015) Crystal structure of the death effector domains of caspase-8. Biochem Bioph Res Co 463, 297-302

46. Vickers CJ, González-Páez GE and Wolan DW (2013) Selective detection of caspase-3 versus caspase-7 using activity-based probes with key unnatural amino acids. ACS Chem Biol 8, 1558-1566

47. Jong WY, Jeffrey PD and Shi Y (2009) Mechanism of procaspase-8 activation by c-FLIPL. Proc Natl Acad Sci USA 106, 8169-8174

48. Schleich K, Warnken U, Fricker N, et al (2012) Stoichiometry of the CD95 death-inducing signaling complex: experimental and modeling evidence for a death effector domain chain model. Mol Cell 47, 306-319

49. Dickens LS, Boyd RS, Jukes-Jones R, et al (2012) A death effector domain chain DISC model reveals a crucial role for caspase-8 chain assembly in mediating apoptotic cell death. Mol Cell 47, 291-305

50. Schleich K, Buchbinder JH, Pietkiewicz S, et al (2015) 
Molecular architecture of the DED chains at the DISC: regulation of procaspase- 8 activation by short DED proteins c-FLIP and procaspase-8 prodomain. Cell Death Differ (advance online publication, 23 October), e1-e16

51. Hoffmann JC, Pappa A, Krammer PH and Lavrik IN (2009) A new C-terminal cleavage product of procaspase-8, p30, defines an alternative pathway of procaspase-8 activation. Mol Cell Boil 29, 4431-4440

52. Golks A, Brenner D, Schmitz I, et al (2006) The role of CAP3 in CD95 signaling: new insights into the mechanism of procaspase-8 activation. Cell Death Differ 13, 489-498

53. Öztürk S, Schleich K and Lavrik IN (2012) Cellular FLICE-like inhibitory proteins (c-FLIPs): fine-tuners of life and death decisions. Exp Cell Res 318, 1324-1331

54. Fricker N, Beaudouin J, Richter P, Eils R, Krammer PH and Lavrik IN (2010) Model-based dissection of CD95 signaling dynamics reveals both a pro-and antiapoptotic role of c-FLIPL. J Cell Biol 190, 377-389

55. Aggarwal BB, Moffat B and Harkins RN (1984) Human lymphotoxin. Production by a lymphoblastoid cell line, purification, and initial characterization. J Biol Chem 259, 686-691

56. Aggarwal BB, Kohr WJ, Hass PE, et al (1985) Human tumor necrosis factor. Production, purification, and characterization. J Biol Chem 260, 2345-2354

57. Locksley RM, Killeen N and Lenardo MJ (2001) The TNF and TNF receptor superfamilies: integrating mammalian biology. Cell 104, 487-501

58. Croft M (2003) Co-stimulatory members of the TNFR family: keys to effective T-cell immunity? Nat Rev Immunol 3, 609-620

59. Idriss HT and Naismith JH (2000) TNF $\alpha$ and the TNF receptor superfamily: Structure-function relationship (s). Microsc Res Tech 50, 184-195

60. Wajant H, Pfizenmaier K and Scheurich P (2003) Tumor necrosis factor signaling. Cell Death Differ 10, 45-65

61. Zhang G (2004) Tumor necrosis factor family ligand-receptor binding. Curr. Opinion Struct Biol 14, 154-160

62. Ea CK, Deng L, Xia ZP, Pineda G and Chen ZJ (2006) Activation of IKK by TNF $\alpha$ requires site-specific ubiquitination of RIP1 and polyubiquitin binding by NEMO. Mol Cell 22, 245-257

63. Vanlangenakker N, Bertrand MJM, Bogaert $P$, Vandenabeele P and Berghe TV (2011) TNF-induced necroptosis in
L929 cells is tightly regulated by multiple TNFR 1 complex I and II members. Cell Death Dis 2, e230

64. Rahighi S, Ikeda F, Kawasaki M, et al (2009) Specific recognition of linear ubiquitin chains by NEMO is important for NF-kB activation. Cell 136, 1098-1109

65. Li J, McQuade T, Siemer AB, et al (2012) The RIP1/RIP3 necrosome forms a functional amyloid signaling complex required for programmed necrosis. Cell 150, 339-350

66. Moulin M, Anderton H, Voss AK, et al (2012) IAPs limit activation of RIP kinases by TNF receptor 1 during development. Embo J 31, 1679-1691

67. Park YH, Jeong MS and Jang SB (2014) Death domain complex of the TNFR-1, TRADD, and RIP1 proteins for death-inducing signaling. Biochem Bioph Res Co 443, 1155-1161

68. Yamaoka S, Courtois G, Bessia C, et al (1998) Complementation cloning of NEMO, a component of the I $\mathrm{B}$ kinase complex essential for NF- $\mathrm{KB}$ activation. Cell 93, 1231- 1240

69. Moquin D and Chan FKM (2010) The molecular regulation of programmed necrotic cell injury. Trends Biochem Sci 35, 434-441

70. Zhang DW, Shao J, Lin J, et al (2009) RIP3, an energy metabolism regulator that switches TNF-induced cell death from apoptosis to necrosis. Science 325, 332-336

71. Hsu H, Shu HB, Pan MG and Goeddel DV (1996) TRADD-TRAF2 and TRADD-FADD interactions define two distinct TNF receptor 1 signal transduction pathways. Cell 84, 299-308

72. Park YH, Jeong MS, Park HH and Jang SB (2013) Formation of the death domain complex between FADD and RIP1 proteins in vitro. BBA-Proteins. Proteom 1834, 292300

73. Jang MS, Lee SJ, Kim CJ, Lee CW and Kim E (2011) Phosphorylation by polo-like kinase 1 induces the tumor-suppressing activity of FADD. Oncogene 30, 471481

74. Kelley SK, Harris LA, Xie D, et al (2001) Preclinical studies to predict the disposition of Apo2L/tumor necrosis factor-related apoptosis-inducing ligand in humans: characterization of in vivo efficacy, pharmacokinetics, and safety. J Pharmacol Exp Ther 299, 31-38

75. El-Deiry WS (2001) Insights into cancer therapeutic design based on p53 and TRAIL receptor signaling. Cell death differ 8, 1066-1075 